\title{
Development of Hexagonal MMR Based UWB Bandpass Filter with 5 GHz Notch Band
}

\author{
Kaliyavarathan Thirumalaivasan, Rangaswamy Nakkeeran \\ Department of Electronics and Communication Engineering, Pondicherry Engineering College, Puducherry, India. \\ Email: thirumalaivasank@pec.edu, rnakeeran@pec.edu
}

Received June $7^{\text {th }}$, 2011; revised July $6^{\text {th }}, 2011$; accepted July $18^{\text {th }}, 2011$.

\begin{abstract}
This paper presents an experimental verification of ultra-wideband bandpass filter (BPF) for UWB applications and notch filter in order to suppress $5 \mathrm{GHz}$ narrowband service when it coexists with UWB radio system. The BPF consists of a hexagonal shaped multiple mode resonator (MMR) with interdigital coupling at both sides. Notch filter is derived from BPF by introducing four embedded open stubs near by the MMR. The developed BPF has insertion loss of $-2 d B$ and the minimum return loss about $-35 d B$, while for the notch filter they are $-3 d B$ and $-40 \mathrm{~dB}$ respectively. The group delay obtained for bandpass filter is below $0.2 \mathrm{~ns}$ and for notch filter, it is about $0.3 \mathrm{~ns}$. With the above structural features the overall dimensions of the filter is $38 \mathrm{~mm}$ (length) $\times 3.2 \mathrm{~mm}$ (breadth) $\times 1.6 \mathrm{~mm}$ (height) and the percentage fractional bandwidth (FBW) of the proposed filter is about $120.48 \%$.
\end{abstract}

Keywords: Ultra-Wideband, MMR, Bandpass Filter, Notch Band, Narrowband, Microstrip

\section{Introduction}

A UWB wireless system uses signal with a low spectrum density in the bandwidth of $3.1-10.6 \mathrm{GHz}$ and transmits the signal at a high transmission speed of 100 - 480 Mbps [1]. The maximum distance between the UWB systems to communicate with each other is about $10 \mathrm{~m}$, and UWB system enables transmission at a higher speed than the conventional WLAN [2]. The filters are one of the primary and necessary components of a microwave system. Microstrip line is a good entrant for filter design due to its advantages of low cost, compact-size, light weight, planar structure and easy integration with other components on a single circuit board. Owing to the low energy per pulse, UWB systems are prone to high level narrow-band interferences (NBI) even though UWB systems may enjoy a high spreading gain due to the large bandwidth. Specifically, IEEE 802.11a system operates around $5 \mathrm{GHz}$, which overlaps the band of UWB signals regulated by the FCC, and can bring significant interference to UWB systems. If such interference is not suppressed properly, the UWB receiver will be jammed. For such circumstances, a UWB filter is required to provide rejection capability in a band from 5 to $6 \mathrm{GHz}$ [3-6].

One solution to this specification is to employ openended quarter-wavelength transmission lines, which provide appropriate rejection at the specific frequency range
$[7,8]$. Conventional UWB filter's designs have been discussed many literatures [9-11]. In the literature [12-15] the simulation, design, fabrication and measurement of UWB bandpass filters and notch filters have been discussed using different geometry like rectangular, circular, ring etc. However, developing such a filter is definitely a challenging work. The first challenge is to achieve $110 \%$ fractional bandwidth requirement, which is used for broadband design. Second comes in the form of requirement of the small and flat group delay over the band.

The proposed and developed UWB bandpass and notch filters, in this paper, are designed with a compact hexagonal MMR. The filters are distinctive in their structure and they have simple design with less number of design parameters compared to the existing filter designs in the literature [16,17]. The paper is organized as follows: Section 2 brings out UWB bandpass filter design. Section 3 explains UWB notch filter design. Results and analysis for the notch filter are presented in Section 4. Section 5 concludes the paper.

\section{Proposed UWB Bandpass Filter}

The UWB bandpass filter studied in this paper consists of feed lines, coupling gaps, and the hexagonal structure. Power is coupled into and out of the resonator through feed lines and coupling gaps. If the distance between the 
feed lines and the resonator is large then the coupling gaps do not affect the resonant frequencies of the hexagon.

The multiple coupled line structure is incorporated with MMR to provide wide transmission band and enhance out-of band performance. The interdigital coupling is used instead of direct coupling for the reason of strong coupling and to introduce capacitance effect to obtain flat passband. The interdigital coupled line structure and MMR are designed and implemented to provide bandpass filtering over 3.1 - 10.6 GHz as shown in Figure 1. The filter is fabricated using FR4 substrate with dielectric constant of 4.4, loss tangent of 0.0004 and thickness of $1.6 \mathrm{~mm}$. The parameters for the developed filter are $\mathrm{L}_{1}=$ $8, \mathrm{~L}_{2}=9.8, \mathrm{~L}_{3}=9.2, \mathrm{~g}_{0}=\mathrm{g}_{1}=\mathrm{g}_{2}=\mathrm{g}_{3}=0.3, \mathrm{~d}_{1}=2, \mathrm{~d}_{2}=4$, $\mathrm{W}=3.2$. All dimensions are with the units of $\mathrm{mm}$. The simulated and measured $\mathrm{S}$ parameters of the proposed UWB bandpass filter on microstrip line using hexagonal MMR is shown in Figure 2. It is clear from the response that the proposed filter has low insertion loss of $-2 \mathrm{~dB}$ and the minimum return loss of about $-35 \mathrm{~dB}$. The fabricated filter has wide passband $2.1-10.4 \mathrm{GHz}$ at the -10 $\mathrm{dB}$ transmission level and percentage fractional bandwidth computed from the response is about $120.48 \%$.

The group delay for the filter is shown in Figure 3, which exhibits a flat group delay response below $0.2 \mathrm{~ns}$ over the whole passband. It implies that this proposed UWB filter has a very good linearity of signal transfer as well and ensures the minimum distortion to the input pulse when implemented in the UWB system. Figure 4 shows the simulation result of phase of $S_{21}$ for UWB bandpass filter. The response shows that the phase of $S_{21}$ throughout the passband of presented filter is acceptably linear for UWB applications.

\section{UWB Notch Filter}

To introduce a good rejection performance at WLAN frequency bands four open stubs are embedded at nearby hexagonal MMR. The four folded arms of each Stepped Impedance Resonator (SIR) are coupled to each other to realize inter-coupling between the ports. A structure to realize the notch band is shown in Figure 5, where the hexagonal MMR with its interdigital-coupled conductors is integrated with the microstrip conductors.

The four open stubs nearby MMR introduce a narrow rejection band from $5.3-5.9 \mathrm{GHz}$ in the UWB passband is

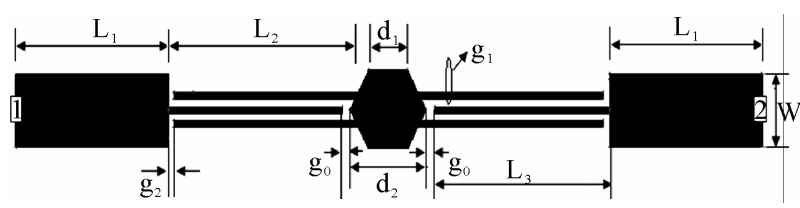

Figure 1. Geometry of the developed bandpass filter.

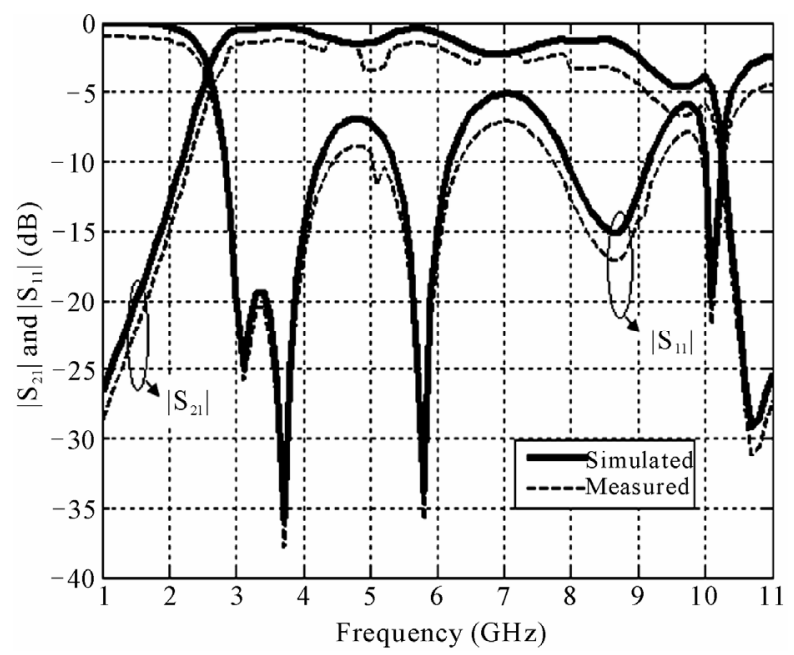

Figure 2. Simulated and measured S parameters of the developed bandpass filter.

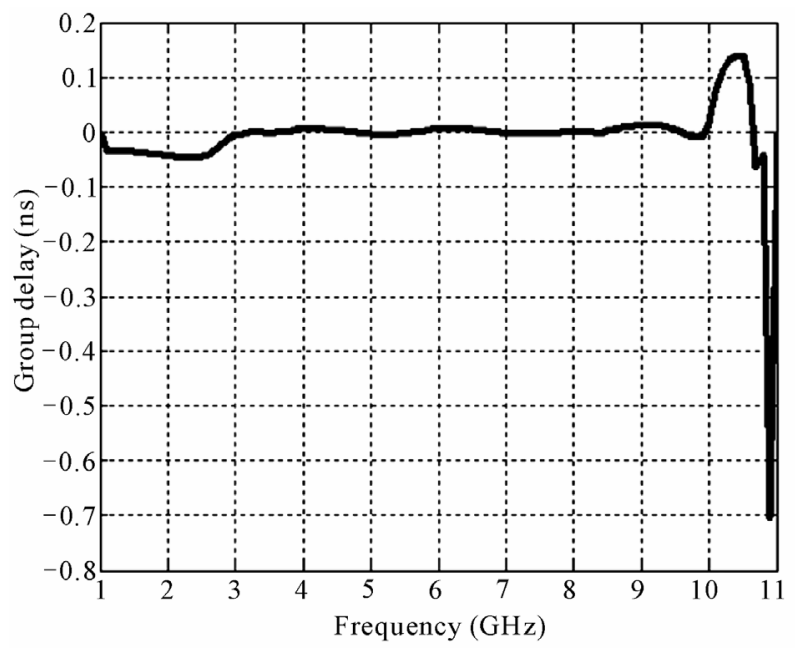

Figure 3. Simulation group delay of bandpass filter.

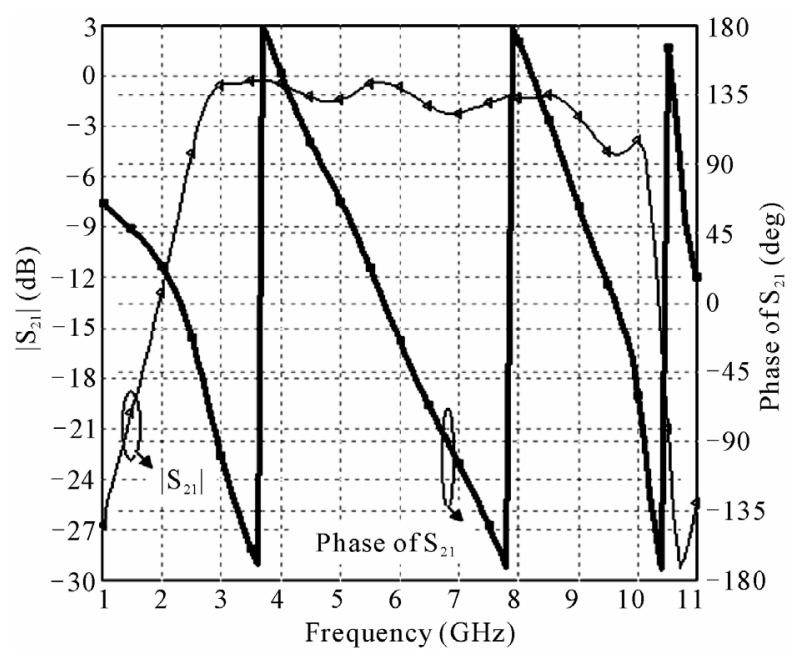

Figure 4. Simulation phase of $S_{21}$ of proposed bandpass filter. 


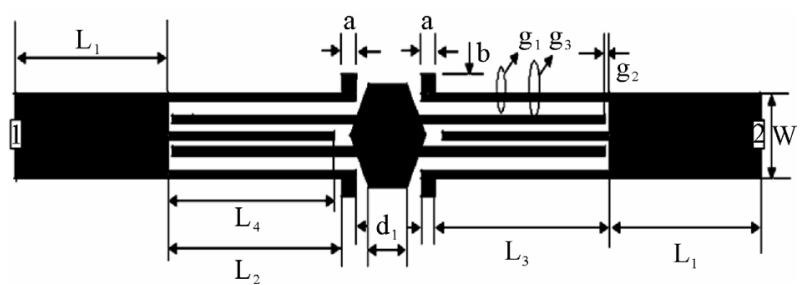

Figure 5. Geometry of the developed UWB notch filter.

witnessed. The notch rejection band can be designed to some specific frequency band(s) by varying the size of the open stubs. The novelty of the proposed filter is introduction of hexagonal shaped MMR to act as a bandpass filter as well as a notch filter for the rejection of WLAN interference. The proposed notch filter is simple, compact and easy to fabricate compare with the existing notch filter designs in the literature.

\section{Results of UWB Notch Filter}

The proposed filter has been fabricated using printed circuit board technology. In order to evaluate the performance of the proposed UWB bandpass filter design, the filter is simulated through the simulation tool IE3D [18]. To analyze the wide band coverage of the filter, the effects of adjusting the filter parameters are investigated.

The simulation and measured S parameters of the developed notch filter is shown in Figure 6. It is clear from the response that the developed filter has better insertion loss of $-3 \mathrm{~dB}$ over both the passbands and the return loss of about $-27 \mathrm{~dB}$. The notch is obtained with linear phase over the passband which can capable of eliminating the influence of IEEE 802.11a. The notch filter proposed here is to trap the upper and lower bands of WLAN exists in the frequency range 5 - $6 \mathrm{GHz}$ from the available passband.

Ensuring the same time differences between signal spectrum components before and after the filtering is very important, especially for wideband signals. This requirement is met if phase response of the filter is linear. Then, the group delay function is constant. Such a condition is enough to assure the linearity of the UWB filter phase response. For the developed UWB notch filter, the computed group delay is less than 0.1 ns which is depicted in Figure 7 and phase response of the same is linear over entire passband as shown in Figure 8.

Filter is designed and fabricated on microstrip substrate FR4 with a dielectric constant of 4.4 and a thickness of $1.6 \mathrm{~mm}$ so that it can be easily integrated with Printed Circuit Board (PCB). Photographs of the fabricated bandpass and notch filters are shown in Figure 9 and Figure 10. As shown in figure, two 50 ohm transmission lines are extended to accommodate the SMA connectors to connect the scalar network analyzer for

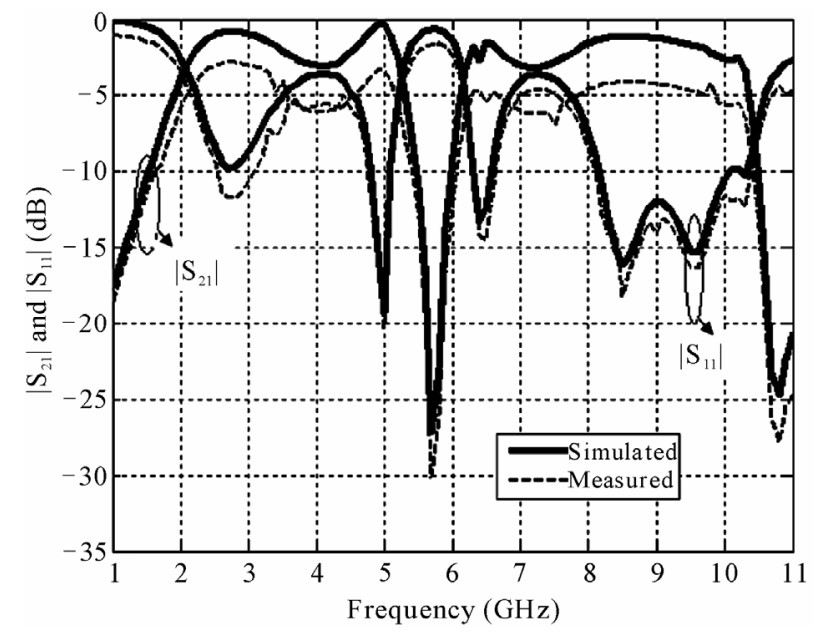

Figure 6. Simulated and measured $S$ parameters of the developed bandpass filter with notch band.

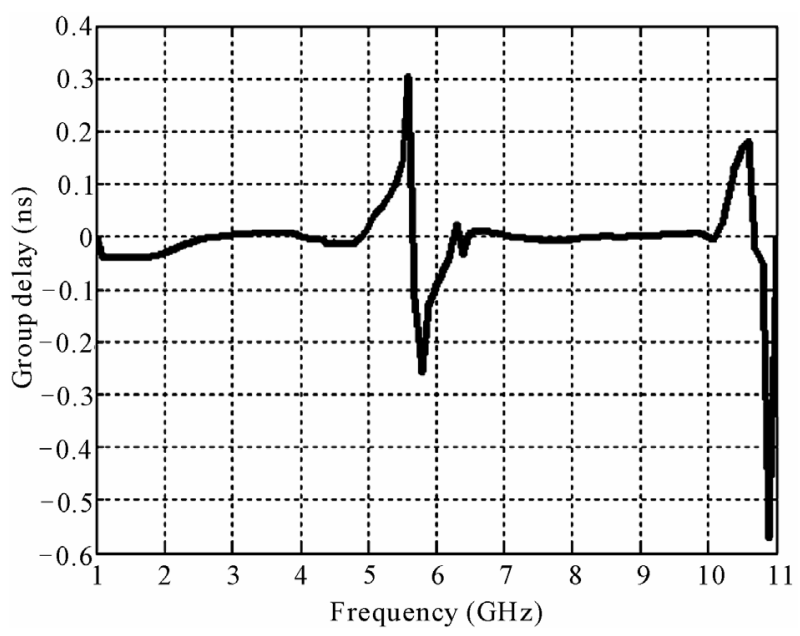

Figure 7. Simulated group delay of UWB bandpass filter with notch band.

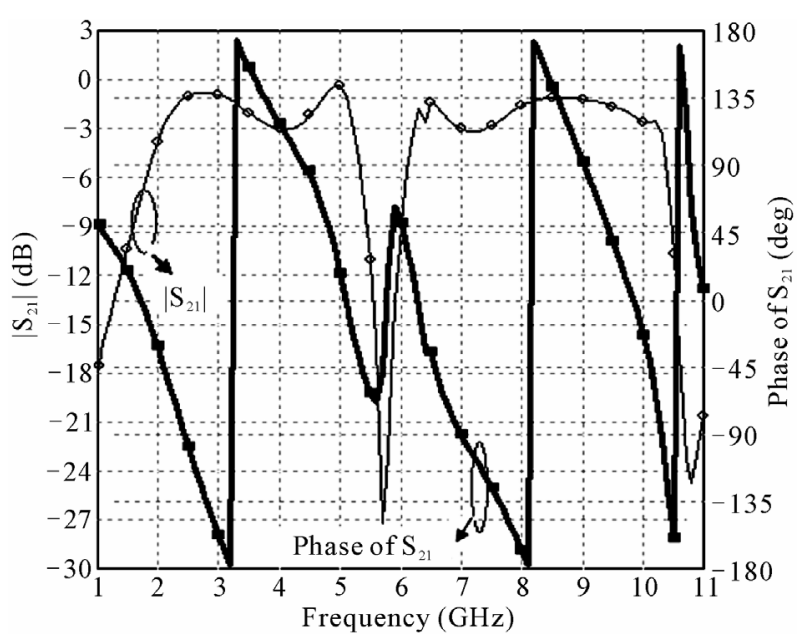

Figure 8. Phase of $S_{21}$ of UWB bandpass filter with notch band. 


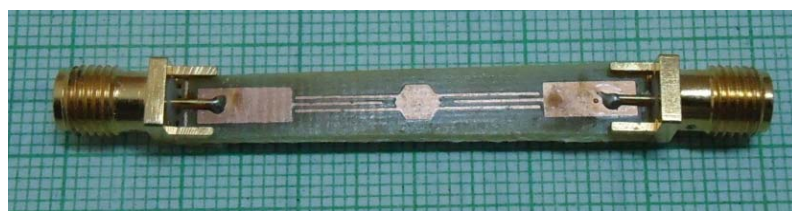

Figure 9. Photograph of the fabricated bandpass filter.

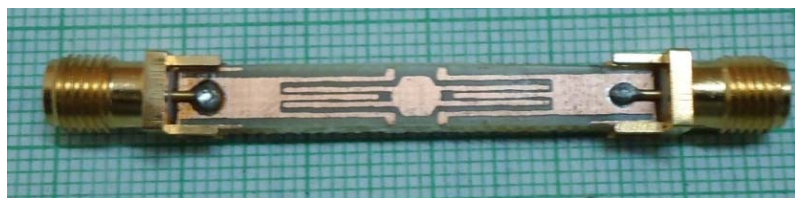

Figure 10. Photograph of the fabricated notch filter.

measurement.

The measured results are comparable with simulation results. The small discrepancy between them might be due to fabrication tolerance, loss tangent of the substrate and parasitic effect of the SMA connectors. Using Hewlett Packard Network analyzer, the insertion loss and return loss of the fabricated filter are measured.

Figure 11 shows the current density distribution on the operating and notch bands. Average E current flow in the network is $14.368 \mathrm{~A} / \mathrm{m}$. For clear understanding, we have given current distribution only for three frames 3.3 $\mathrm{GHz}$ for the lower operating band, $5.5 \mathrm{GHz}$ at the notch band and $8.5 \mathrm{GHz}$ at the upper operating band. It can be observed from the Figures 11(a) and (c), maximum current density distribution occurs at the operating bands. It is clear from the Figure 11(b) that the minimum current distribution is at the rejection band of $5.5 \mathrm{GHz}$.

The developed notch filter is more compact with comparable performances than filters available in the literature. Comparison of some of the filters with respect to their geometry, size and performance is tabulated in Table 1.

\section{Conclusions}

The UWB bandpass filter using hexagonal shaped MMR with $5 \mathrm{GHz}$ notch band is presented in his paper to suppress WLAN. The filter is implemented by integrating hexagonal MMR coupled with interdigital conductors of the filter. The developed BPF demonstrated with excellent ultra-wide passband from $2.2 \mathrm{GHz}$ to $10.5 \mathrm{GHz}$ with an insertion and return loss is about $-2 \mathrm{~dB}$ and $-36 \mathrm{~dB}$ respectively. Rejection performance for the filter is about $-27 \mathrm{~dB}$ at the centre frequency of $5.4 \mathrm{GHz}$ and the filter has good outband performance higher than the requirement of FCC's mask. The group delay obtained for bandpass filter at the operating is about 0.2 ns and for notch filter it is below 0.1 ns. With the above structural features the overall dimensions of the filter are $38 \mathrm{~mm}$ (length) $\times 3.2 \mathrm{~mm}$ (breadth) $\times 1.6$ (height) $\mathrm{mm}$ and the

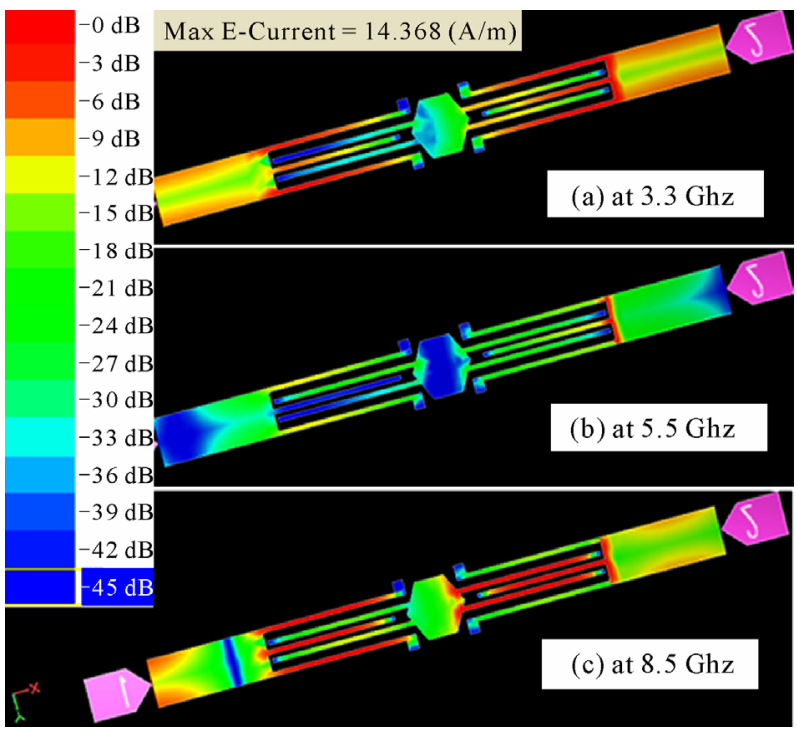

Figure 11. Current distribution for different frames.

Table 1. Comparison of developed filter with best reported UWB bandpass filter with notch band in the literature.

\begin{tabular}{ccccc}
\hline Parameters/Ref. & {$[8]$} & {$[9]$} & {$[10]$} & This work \\
\hline $\mathrm{S}_{21}(\mathrm{~dB})$ & -1.33 & -1.5 & -2.3 & $<-1.43$ \\
$\mathrm{f}_{\mathrm{c}}(\mathrm{GHz})$ & 6.69 & 6.85 & 5.8 & 6.2 \\
FBW (\%) & 102.8 & 113 & 110 & 120.48 \\
Group delay (ns) & $0.29-1.12$ & 0.2 & 0.19 & $<0.1$ \\
Notch at fc (GHz) & 5.41 & 5.5 & 5.8 & 5.7 \\
Rejection Level (dB) & -11.14 & -32.25 & -18.8 & -28 \\
Rejection BW \% & 3.5 & 9.09 & 4.6 & 10.97 \\
\hline
\end{tabular}

fractional bandwidth of the UWB bandpass filter is about $120.48 \%$. The obtained S parameters characteristics of both UWB bandpass and notch filter convey optimal performance in terms of insertion and return loss measurements. Since the frequency where notch occurs is exactly match with center frequency of WLAN, these types of filters are highly suitable for filtering when UWB coexist with other radio systems.

\section{REFERENCES}

[1] Federal Communications Commission (FCC), "Revision of Part 15 of the Commission's Rules Regarding Ultra-Wideband Transmission Systems FCC,” FCC Technical Report, ET-Docket FCC02-48, February 2002, pp. 98-153.

[2] Technical Committee of Home Networks and EMC, "Home Network and EMC," Ohm-Sha, Institute of Electrical Engineers of Japan, Tokyo, 2006.

[3] J. S. Hong, "Microstrip Filters for RF/Microwave Appli- 
cation,” Wiley, New York, 2001. doi:10.1002/0471221619

[4] G. Mattaei, L. Young and E. M. T. Jones, "Microwave Filters, Impedance-Matching Networks, and Coupling Structures,” Artech House, Narwood, 1980.

[5] G.-M. Yang, R. Jin, C. Vittoria, V. G. Harris and N. X. Shun, "Small Ultra-Wideband (UWB) Bandpass Filter with Notched Band," IEEE Microwave Theory and Wireless Component Letters, Vol. 18, No. 3, 2008, pp. 176178. doi:10.1109/LMWC.2008.916781

[6] C.-Y. Hsu, L.-K. Yeh, C.-Y. Chen and H.-R. Chuang, “A 3 - $10 \mathrm{GHz}$ Ultra-Wideband Bandpass Filter with 5 - 6 GHz Rejection Band,” Proceedings of Asia-Pacific Microwave Conference, Bangkok, 11-14 December 2007.

[7] P.-Y. Hsiao and R. M. Weng, "Compact Open Loop UWB Filter with Notch Band," Journal of Progress in Electromagnetic Research Letters, Vol. 7, 2009, pp. 149159. doi:10.2528/PIERL09022501

[8] C. H. Kim and K. Chang, "Ultra-Wideband Ring Resonator Bandpass Filter with a Notched Band," IEEE Microwave and Wireless Components Letters, Vol. 21, No. 4, 2011, pp. 1-3. doi:10.1109/LMWC.2011.2109942

[9] S. S. Karthikeyan and R. S. Kshetrimayum, "Notched UWB Bandpass Filter Using Complementary Single Split Ring Resonator,” IEICE Electron Express, Vol. 7, No. 17, 2010, pp. 1290-1295. doi:10.1587/elex.7.1290

[10] P.-Y. Hsiao and R. M. Weng, "Compact Open Loop UWB Filter with Notch Band,” Progress in Electromagnetics Research Letters, Vol. 7, 2009, pp. 149-159. doi:10.2528/PIERL09022501

[11] M. Meeloon, S. Chaimool and P. Akkaraekthalin, "Broadband Bandpass Filters Using Slotted Resonators Fed by Interdigital Coupled Lines for Improving Upper Stopband
Performances," Proceedings of Asia-Pacific Microwave Conference, Bangkok, 11-14 December 2007, pp. 14051408. doi:10.1109/APMC.2007.4555111

[12] L. Zhu, S. Sun and W. Menzel, "Ultra-Wideband (UWB) Bandpass Filters Using Multiple-Mode Resonator," IEEE Microwave Theory and Wireless Component Letters, Vol. 15, No. 11, 2005, pp. 796-798. doi:10.1109/LMWC.2005.859011

[13] K. Thirumalaivasan, R. Nakkeeran and S. Oudayacoumar, "Development of UWB Dual Notch Filter to Reject IEEE 802.11a Using Ring Resonator," Proceedings of IEEE International Conference on WOCN, Colombo, 6-8 September 2010

[14] Q. Li, C.-H. Liang, H.-B. Wen and G.-C. Wu, "Compact Planar Ultra-Wideband (UWB) Bandpass Filter with Notched Band,” Proceedings of Asia-Pacific Microwave conference, München, 16-18 March 2009.

[15] H. Shaman and J.-S. Hong, "An Asymmetric Parallel Coupled Line for Notch Implementation in UWB Filters," IEEE Microwave Theory and Wireless Component Letters, Vol. 17, No. 7, 2007, pp. 516-518. doi:10.1109/LMWC.2007.899314

[16] K. Thirumalaivasan, R. Nakkeeran and S. Oudayacoumar, "Development of UWB Bandpass Filter Using Ring Resonator," Proceedings of IEEE International Conference on Ultra-wideband (ICUWB), Nanjing, 23-27 September 2010.

[17] X. W. Shi, X. H. Wang and Q. L. Huang, "Compact UWB Bandpass Fillter with Notched Band,” Journal of Progress in Electromagnetic Research, Vol. 4, 2008, pp. 121-128.

[18] IE3D 14, Zeland Software, Ins., Fremont, USA. 